\title{
Role of a new bioassay for thyroid-stimulating antibodies (aequorin TSAb) in Graves' ophthalmopathy
}

\author{
Yuji Hiromatsu' ${ }^{1), 2)}$, Hiroyuki Eguchi ${ }^{1)}$, Yuko Matsuo ${ }^{1)}$, Tamotsu Kato ${ }^{1)}$, Junichi Tani1),3), Shiho Watanabe ${ }^{4)}$, \\ Yasuo Teshima ${ }^{4)}$ and Naohiro Araki ${ }^{5)}$ \\ 1) Division of Endocrinology and Metabolism, Department of Medicine, Kurume University School of Medicine, Kurume, Fukuoka \\ 830-0011, Japan \\ 2) Thyroid Center, Shin Koga Hospital, Kurume, Fukuoka 830-8577, Japan \\ 3) Division of Nephrology and Endocrinology, Faculty of Medicine, Tohoku Medical and Pharmaceutical University, Sendai, Miyagi \\ 983-8536, Japan \\ 4) Department of Ophthalmology, Kurume University School of Medicine, Kurume, Fukuoka 830-0011, Japan \\ ${ }^{5)}$ Diagnostic Division, Otsuka Pharmaceutical Co. Ltd., Tokyo 108-8242, Japan
}

\begin{abstract}
Graves' ophthalmopathy (GO) is characterized by an autoimmune reaction against thyrotropin (TSH) receptors and is diagnosed by TSH receptor antibody (TRAb). A novel assay for thyroid-stimulating antibody (TSAb) was recently introduced using a frozen Chinese hamster ovary cell line expressing TSH receptors, cyclic adenosine monophosphate (cAMP)-gated calcium channel, and aequorin (aequorin TSAb). The aim of this study was to evaluate the role of aequorin TSAb in GO. We studied 136 Japanese patients with GO (22 euthyroid and 8 hypothyroid GO patients) at our hospital. TRAbs were estimated by first generation TRAb (TRAb $1^{\text {st }}$ ), second generation TRAb (hTRAb $2^{\text {nd }}$ ), conventional porcine TSAb, and the new aequorin TSAb assays. Aequorin TSAb, porcine TSAb, TRAb $1^{\text {st }}$, and hTRAb $2^{\text {nd }}$ were positive in $125 / 136(92 \%)$, 110/136 (81\%), 81/130 (62\%), and 93/114 (82\%) patients, respectively. In patients with hyperthyroid GO, they were positive in 98/106 (98\%), 96/106 (91\%), 78/101 (77\%), and 84/93 (90\%) patients, respectively. In patients with euthyroid GO, they were positive in 19/22 (86\%), 9/22 (41\%), $1 / 21(5 \%)$, and 6/17 (35\%) patients, respectively. Aequorin TSAb levels were significantly related to TRAb $1^{\text {st }}(r=0.4172, p<0.0001)$, hTRAb $2^{\text {nd }}(r=0.2592, p<0.0001)$, and porcine TSAb $(r=0.4665$, $p<0.0001)$. Clinical activity score (CAS) was significantly greater in patients with high titers of aequorin TSAb than in those with low titers. Aequorin TSAb levels were significantly related to the signal intensity ratio of the enlarged eye muscle and proptosis evaluated by MRI before steroid pulse therapy. Aequorin TSAb assay was more sensitive than the conventional assays, especially in euthyroid GO.
\end{abstract}

Key words: Graves' ophthalmopathy, Thyrotropin receptor antibody, Thyroid-stimulating antibody, Aequorin

GRAVES' OPHTHALMOPATHY (GO) is an autoimmune disorder characterized by autoimmunity against the thyrotropin (TSH) receptor in the orbit and is closely associated with autoimmune thyroid diseases. GO is clinically manifested in $25-50 \%$ of patients with Graves' disease and in $2 \%$ of patients with chronic thyroiditis [1-4]. At the onset of ophthalmopathy, $80-90 \%$ of GO patients have hyperthyroidism, while the rest have euthyroidism or hypothyroidism without any history of Graves' hyperthyroidism, and are called as euthyroid GO [5] and hypothyroid GO [6], respectively. Although the

Submitted Sep. 5, 2019; Accepted Nov. 11, 2019 as EJ19-0398 Released online in J-STAGE as advance publication Dec. 10, 2019 Correspondence to: Yuji Hiromatsu, MD, PhD., Thyroid Center, Shin Koga Hospital, 120 Tenjin-cho, Kurume, Fukuoka 830-8577, Japan.

E-mail: yuji@med.kurume-u.ac.jp primary autoantigen(s) and precise mechanisms involved in the association of GO with autoimmune thyroid diseases are still unclear, TSH receptor present in the orbital tissues is thought to be the primary target of autoimmune reactions in GO [2, 7]. The TSH receptor antibodies (TRAbs) have been reported to be associated with the development of GO [8-10]. Further, thyroid stimulating immunoglobulins (TSI) were found to be directly associated with clinical activity score (CAS) and response to anti-inflammatory therapy $[10,11]$. The TRAbs, therefore, are used for the diagnosis and management of GO. Recently, a novel assay for thyroid-stimulating antibody (TSAb) has been introduced using a genetically engineered Chinese hamster ovary $(\mathrm{CHO})$ cell line expressing the human TSH receptor, cyclic adenosine monophosphate (cAMP)-gated calcium channel, and aequorin (aequorin TSAb) [12]. It seems to be more sen- 
sitive than the conventional assays for Graves' disease [12]. The aim of this study was to evaluate the role of aequorin TSAb in GO.

\section{Materials and Methods}

We studied 136 Japanese patients with GO (mean age 53 yr, range $27-81$ yr, 86 females) who visited the Kurume University Hospital and Kurume University Medical Center between 2011 and 2015. At the time of diagnosis of ophthalmopathy, 106 patients had hyperthyroidism, 22 were euthyroid (euthyroid GO), and 8 had hypothyroidism without any history of hyperthyroidism (hypothyroid GO). Among them, fifty patients received methylprednisolone pulse therapy for moderate to severe, or sight-threatening and active GO and were followed for $>1$ year in our hospital. Ophthalmological examinations and magnetic resonance imaging (MRI) were performed at baseline, and at 1,6 , and 12 months after pulse therapy, as previously described $[13,14]$.

Informed consent was obtained from the patients and the ethical approval was obtained from the Ethical Committee of Kurume University.

\section{Laboratory assays}

TRAb estimation was performed by the first generation TRAb (TRAb 1 ${ }^{\text {st }}$, TRAb Cosmic III kit, Cosmic Co., Japan) with a cutoff value of $10 \%$, the second generation assay (hTRAb 2 $2^{\text {nd; }}$ DYNO test TRAb Human kit 'Yamasa'; Yamasa Co., Choshi, Chiba, Japan) with a cutoff value of $1.0 \mathrm{IU} / \mathrm{L}$, the conventional TSAb kit 'Yamasa' (Yamasa) with a cutoff value of $180 \%$, and the new aequorin TSAb assay (Otsuka Pharmaceutical Co., Ltd. Minato-ku, Tokyo, Japan) with a cutoff value of 35 $\mathrm{mIU} / \mathrm{L}$, as previously reported [12].

\section{Ophthalmological examinations}

Ophthalmological examinations were performed by two expert ophthalmologists for GO using the modified NOSPECS classification [15] and CAS [16]. Proptosis was assessed with a Hertel exophthalmometer. The ocular movements were evaluated with a Hess chart. The alterations of eye symptoms were recorded based on an ophthalmopathy index according to the American Thyroid Association classification for eye sign changes in GO. The ophthalmopathy index was calculated as the sum of the scores $(0-3)$ for each class ranging from 0 to 15 [17]. The CAS was graded according to the modified CAS system developed by the European Group on Graves' orbitopathy (EUGOGO) [16]. The quality of life was evaluated using the scores developed by EUGOGO [16].

\section{Magnetic resonance imaging}

MRI of the orbits was performed in the parasagittal, horizontal, and coronal planes with a 3.0-T superconducting MR unit (MAGNETOM Skyra, Siemens) having a surface coil (eye coil). The thickness of the superior, medial, and inferior rectus muscle was estimated at the site of their enlargement in the coronal section of the T1weighted image. The thickness of the lateral rectus muscle was estimated at the site of its enlargement in a horizontal section of the T1-weighted image. The enlargement of the eye muscle was also expressed as a ratio of the thickness of the eye muscle to the diameter of the optic nerve to avoid individual variation. The signal intensity of the enlarged ocular muscle was also measured at the site of enlargement in the Short T1 Inversion Recovery (STIR) image and was compared to that of the cerebral substantia alba, expressing as the signal intensity ratio [14]. Proptosis was also determined as the distance between the corneal eminence and the line connecting the two zygomatic bones in a horizontal section of the T1-weighted image. Muscle measurement was performed on the same muscle and the same section.

\section{Protocol of the steroid pulse therapy}

Methylprednisolone was administered for 3 days at a daily dosage of 0.5 or $1 \mathrm{~g}$ diluted in $500 \mathrm{~mL}$ isotonic saline infused intravenously over $2 \mathrm{~h}$ [18]. The patients were treated thrice repeatedly. Ophthalmological examination was performed within one week after the last cycle of pulse therapy. Ophthalmological examinations and MRI were also performed at 6 and 12 months after the pulse therapy, as previously described.

\section{Statistical analysis}

The continuous variables were compared between the two groups using the $t$-test or Wilcoxon rank-sum test, while the categorical variables were analyzed using the chi-square test or Fisher's exact probability test. The relationship between aequorin TSAb and porcine TSAb or TRAbs was examined by linear correlation analysis. A $p$-value $<0.05$ was considered statistically significant. All statistical analyses were performed using JMP software (JMP ${ }^{\circledR}$ pro12.2.0, SAS Institute Inc., SAS Campus Drive, Cary, NC, USA).

\section{Results}

\section{Prevalence of anti-TSH receptor antibody in Graves, ophthalmopathy}

The aequorin TSAb, conventional porcine TSAb, TRAb $1^{\text {st }}$, and hTRAb $2^{\text {nd }}$ were positive in $125 / 136$ (92\%), 110/136 (81\%), 81/130 (62\%), and 93/114 (82\%) patients with ophthalmopathy, respectively (Table 1). 
Table 1 Prevalence of anti-TSH receptor antibodies in patients with Graves' ophthalmopathy $(N=136)$.

\begin{tabular}{lcccc}
\hline \multirow{2}{*}{ Normal range } & Aequorin TSAb & Porcine TSAb & TRAb 1st & hTRAb 2nd \\
\cline { 2 - 4 } & $<35 \mathrm{mIU} / \mathrm{L}$ & $<180 \%$ & $<15 \%$ & $<1.0 \mathrm{IU} / \mathrm{L}$ \\
\hline Hyperthyroid GO & $98 / 106(98 \%)$ & $96 / 106(91 \%)$ & $78 / 101(77 \%)$ & $84 / 93(90 \%)$ \\
Euthyroid GO & $19 / 22(86 \%)$ & $9 / 22(41 \%)^{* * *}$ & $1 / 21(5 \%)^{* * * *}$ & $6 / 17(35 \%)^{* * *}$ \\
Hypothyroid GO & $8 / 8(100 \%)$ & $5 / 8(63 \%)$ & $2 / 8(25 \%)$ & $3 / 4(75 \%)$ \\
Total & $125 / 136(92 \%)$ & $110 / 136(81 \%)^{* *}$ & $81 / 130(62 \%)^{* * * *}$ & $93 / 114(82 \%)^{*}$ \\
\hline
\end{tabular}

${ }^{*} p<0.05,{ }^{* *} p<0.01, * * * p<0.005, * * * * p<0.001$.
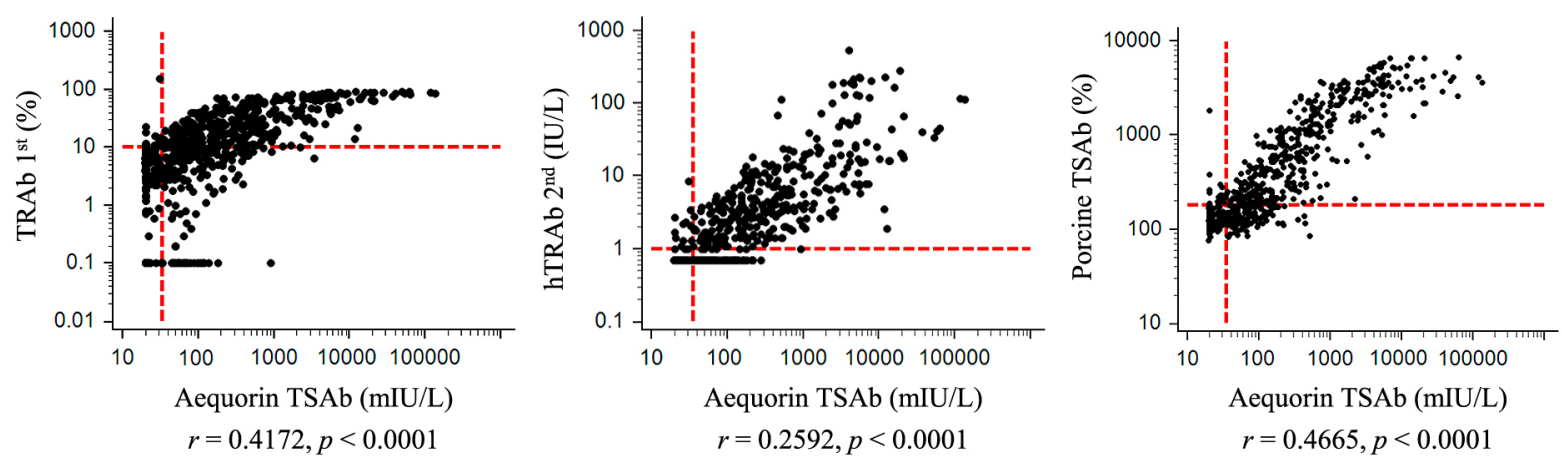

Fig. 1 Correlation between aequorin TSAb and TRAb $1^{\text {st }}$, human TRAb $2^{\text {nd }}$ and porcine TSAb. The dotted lines represent manufacturer's cutoff lines of the assay kits.

Table 2 Aequorin TSAb and ophthalmological examinations before pulse therapy $(N=50)$.

\begin{tabular}{lccccccc}
\hline $\begin{array}{l}\text { Aequorin TSAb } \\
(\mathrm{mIU} / \mathrm{L})\end{array}$ & $\begin{array}{c}\text { Male/ } \\
\text { Female }\end{array}$ & Age (years) & $\begin{array}{c}\text { Smoking } \\
\text { yes/no }\end{array}$ & CAS & NOSPECS scores & $\begin{array}{c}\text { Proptosis right eye } \\
(\mathrm{mm})\end{array}$ & $\begin{array}{c}\text { Proptosis left eye } \\
(\mathrm{mm})\end{array}$ \\
\hline$\geqq 454.5$ & $7 / 18$ & $56.1 \pm 13.2$ & $9 / 16$ & $3.5 \pm 1.6$ & $6.4 \pm 2.1$ & $19.6 \pm 2.9$ & $19.9 \pm 3.8$ \\
$<454.5$ & $5 / 20$ & $52.2 \pm 15.2$ & $8 / 17$ & $2.1 \pm 1.4$ & $5.7 \pm 1.9$ & $18.0 \pm 2.5$ & $18.4 \pm 2.6$ \\
$p$-value & & 0.35 & & 0.002 & 0.26 & 0.10 & 0.10 \\
\hline
\end{tabular}

The positive ratio in aequorin TSAb assay was significantly greater than that in conventional porcine TSAb, TRAb $1^{\text {st }}$, or TRAb $2^{\text {nd }}$ generation assay. In patients with euthyroid GO, aequorin TSAb, conventional porcine TSAb, TRAb $1^{\text {st }}$, and hTRAb $2^{\text {nd }}$ were positive in $19 / 22$ (86\%), 9/22 (41\%), 1/21 (5\%), and 6/17 (35\%) patients, respectively. The positive ratio in aequorin TSAb assay was significantly greater than that in conventional porcine TSAb, TRAb $1^{\text {st }}$, or TRAb $2^{\text {nd }}$ generation assay.

\section{Correlation between aequorin TSAb and TRAb $1^{\text {st }}$, and human TRAb and porcine TSAb}

The aequorin TSAb levels were significantly related to the TRAb $1^{\text {st }}$, hTRAb $2^{\text {nd }}$, and porcine TSAb levels (Fig. $1)$.

\section{Relationship between aequorin TSAb and ophthalmological examinations}

CAS was significantly greater in patients with high ti- ters of aequorin TSAb than in those with low titers (Table 2). Proptosis tended to be greater in patients with high titers of aequorin TSAb.

\section{Relationship between aequorin TSAb and orbital findings before pulse therapy for $G O$}

The aequorin TSAb levels were significantly related to the signal intensity ratio of the enlarged eye muscle and proptosis evaluated by MRI before steroid pulse therapy (Fig. 2).

\section{Relationship between aequorin TSAb and changes in orbital findings assessed by MRI after pulse therapy $(N=50)$}

Significant improvements in the size and signal intensity ratio of the enlarged extraocular muscles were observed in GO patients with an either high or low titer of aequorin TSAb (Table 3). The improvement persisted up to 1 year. In contrast, proptosis and retro-orbital vol- 

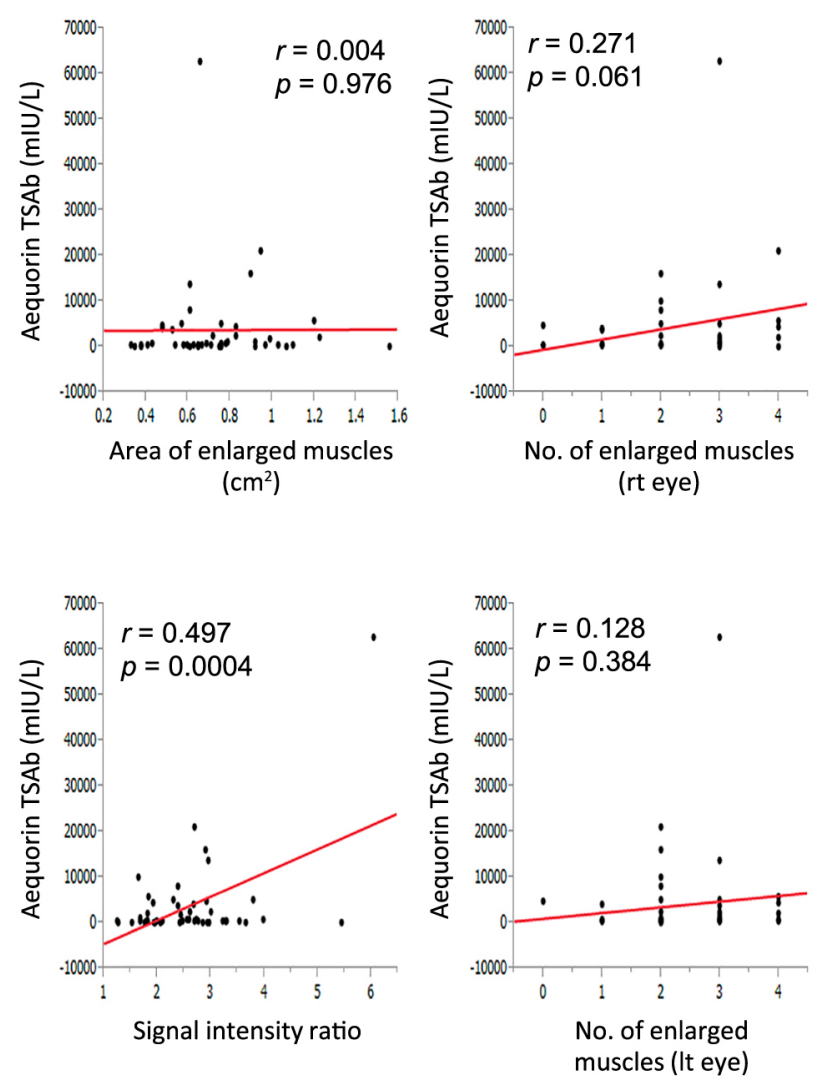
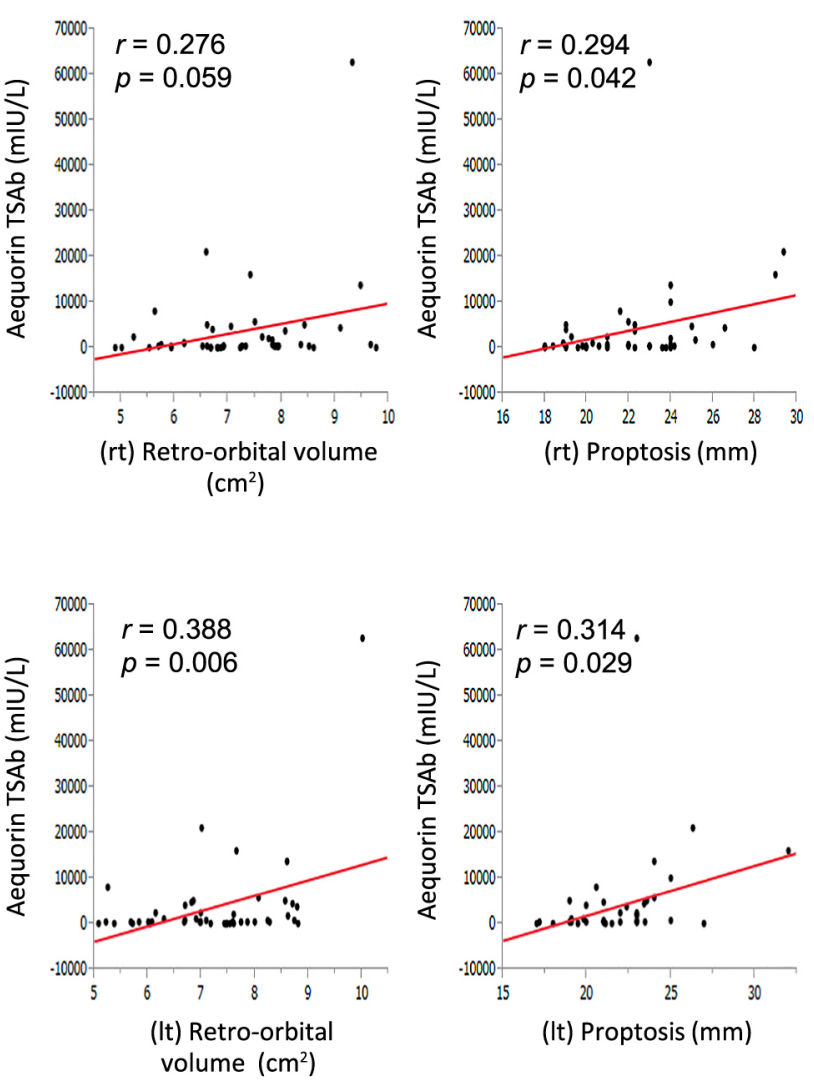

Fig. 2 Relationship between aequorin TSAb and orbital findings before pulse therapy for GO $(N=50)$

ume were improved in GO patients with high titers of aequorin TSAb at 1 month after the pulse therapy, but the values exacerbated at 6 months after the pulse therapy.

\section{Discussion}

In the present study, the highest sensitivity of aequorin $\mathrm{TSAb}$ was obtained in GO, especially in euthyroid GO (86\%), whereas that of porcine TSAb, TRAb $1^{\text {st }}$, and hTRAb $2^{\text {nd }}$ were $41 \%, 5 \%$, and $35 \%$, respectively. These results suggest the usefulness of aequorin TSAb for early diagnosis, making the decision for treatment, and monitoring treatment in GO, especially in euthyroid GO. A previous study showed that aequorin TSAb assay has wider ranges in the measurement and have a shorter assay time [13]. Aequorin TSAb assay directly detects intra-cellular cAMP increase via cyclic nucleotide-gated ion channels on the cell membrane without homogenization, which may increase its sensitivity. The intra-assay and interassay variation in aequorin TSAb assay was low enough to be acceptable for the routine use [13]. Furthermore, it does not require a sterilized culture system. This assay, therefore, may be beneficial for use in the general clinical laboratories.

There are several reports demonstrating the association of TRAbs with ophthalmopathy. Noh et al. reported that the severity of ophthalmopathy was associated with TSAb but not thyrotropin-binding inhibitor immunoglobulin (TBII) [8]. Eckstein et al. showed a positive association of the second-generation TBII assay based on the human recombinant TSH receptor (hTRAb $2^{\text {nd }}$ ) with the severity of ophthalmopathy [9]. Lytton et al. reported thyroid stimulating immunoglobulin (TSI) bioassay in a cross-sectional study using $\mathrm{CHO}$ cell expressing the chimeric human TSH receptor, which had amino acids 262 to 335 substituted with 73 amino acids from the rat $\mathrm{LH}$ receptor (Mc4/CHO) [10]. They found that TSI was positive in $97 \%$ of GO, and the TSI level correlated with the CAS. They suggested that TSI is a clinically useful tool for the management of patients with GO [11]. Furthermore, the present study showed the positive association between the aequorin TSAb levels and orbital findings quantitatively assessed by MRI. The proptosis and enlargement of eye muscles assessed by MRI were greater in patients having a high titer of aequorin TSAb. These results suggest the aequorin TSAb level may be a promising biomarker for GO.

This study has some limitations. First, it was a crosssectional study and retrospective in design. There was not adequate data related to the outcome of ophthalmopathy post therapy. Second, the number of included patients was small, which also prevented us from arriv- 
Table 3 Relationship between aequorin TSAb and changes in the orbital findings assessed by MRI after pulse therapy.

\begin{tabular}{|c|c|c|c|c|}
\hline & \multicolumn{3}{|c|}{ Aequorin TSAb (mIU/L) } & \multirow{2}{*}{$p$-value ${ }^{\S}$} \\
\hline & total & $\geqq 454.5$ & $<454.5$ & \\
\hline \multicolumn{5}{|c|}{ Area of the enlarged muscle } \\
\hline before & $0.73 \pm 019$ & $0.75 \pm 0.21$ & $0.71 \pm 0.28$ & 0.580 \\
\hline 1 month & $0.54 \pm 0.12 * * * *$ & $0.58 \pm 0.16 * * * *$ & $0.50 \pm 0.15 * * * *$ & 0.075 \\
\hline 6 months & $0.55 \pm 0.17 * * * *$ & $0.60 \pm 0.23 * * * *$ & $0.48 \pm 0.17 * * *$ & 0.118 \\
\hline 1 year & $0.50 \pm 0.13 * * * *$ & $0.53 \pm 0.17 * * * *$ & $0.45 \pm 0.12 * *$ & 0.116 \\
\hline \multicolumn{5}{|c|}{ Signal intensity area } \\
\hline before & $2.60 \pm 0.66$ & $2.69 \pm 0.92$ & $2.54 \pm 0.90$ & 0.471 \\
\hline 1 month & $2.09 \pm 0.53 * * * *$ & $2.17 \pm 0.64^{*}$ & $2.07 \pm 0.70 * * *$ & 0.606 \\
\hline 6 months & $1.60 \pm 0.36^{* * * *}$ & $1.73 \pm 0.45^{* *}$ & $1.44 \pm 0.35^{*}$ & 0.130 \\
\hline 1 year & $1.53 \pm 0.33 * * * *$ & $1.58 \pm 0.40 * * * *$ & $1.40 \pm 0.33 * * * *$ & 0.198 \\
\hline \multicolumn{5}{|c|}{ Proptosis (right eye) } \\
\hline before & $21.8 \pm 2.34$ & $23.0 \pm 2.85$ & $20.8 \pm 2.52$ & 0.007 \\
\hline 1 month & $20.8 \pm 1.69 * * * *$ & $21.2 \pm 1.97 * * * *$ & $20.4 \pm 2.09$ & 0.232 \\
\hline 6 months & $21.6 \pm 2.41$ & $22.9 \pm 2.69$ & $20.4 \pm 2.40$ & 0.076 \\
\hline 1 year & $20.6 \pm 2.00^{* * * *}$ & $20.9 \pm 2.39 * * * *$ & $20.9 \pm 3.09$ & 0.747 \\
\hline \multicolumn{5}{|c|}{ Proptosis (left eye) } \\
\hline before & $21.7 \pm 2.10$ & $23.0 \pm 2.68$ & $20.5 \pm 2.24$ & 0.001 \\
\hline 1 month & $20.7 \pm 1.83^{* * * *}$ & $21.3 \pm 2.38 * * * *$ & $20.2 \pm 2.38$ & 0.120 \\
\hline 6 months & $21.6 \pm 2.32$ & $22.7 \pm 2.75$ & $20.5 \pm 2.40$ & 0.112 \\
\hline 1 year & $20.8 \pm 2.21 * * * *$ & $21.4 \pm 2.71 * * *$ & $20.8 \pm 3.17$ & 0.432 \\
\hline \multicolumn{5}{|c|}{ Retro-orbital volume (right eye) } \\
\hline before & $7.18 \pm 1.22$ & $7.44 \pm 1.26$ & $6.94 \pm 1.12$ & 0.160 \\
\hline 1 month & $6.93 \pm 1.12$ & $7.04 \pm 1.03 * *$ & $6.82 \pm 1.22$ & 0.515 \\
\hline 6 months & $6.83 \pm 0.93$ & $7.25 \pm 0.72 *$ & $6.33 \pm 0.93$ & 0.010 \\
\hline 1 year & $7.02 \pm 1.14$ & $7.22 \pm 0.80^{*}$ & $6.70 \pm 1.51$ & 0.218 \\
\hline \multicolumn{5}{|c|}{ Retro-orbital volume (left eye) } \\
\hline before & $7.21 \pm 1.14$ & $7.54 \pm 1.15$ & $6.90 \pm 1.02$ & 0.050 \\
\hline 1 month & $6.91 \pm 1.12$ & $7.13 \pm 1.22 * * * *$ & $6.68 \pm 0.99 * *$ & 0.164 \\
\hline 6 months & $6.97 \pm 1.05$ & $7.36 \pm 0.95^{* * *}$ & $6.50 \pm 0.99$ & 0.004 \\
\hline 1 year & $7.09 \pm 1.19$ & $7.30 \pm 1.01$ & $6.75 \pm 1.30$ & 0.217 \\
\hline
\end{tabular}

${ }^{*} p<0.05,{ }^{* *} p<0.01,{ }^{* *} p<0.005,{ }^{* * * *} p<0.001$ (before $v s .1$ month, 6 months, and 1 year after pulse therapy) ${ }^{\S} p$-value between patients with aequorin $\mathrm{TSAb} \geqq 454.5 \mathrm{mIU} / \mathrm{L}$ and those with aequorin $\mathrm{TSAb}<454.5 \mathrm{mIU} / \mathrm{L}$.

ing at definite conclusions. Third, there were no functional studies on the pathological mechanisms of GO. However, our studies showed some positive association of aequorin TSAb and the effect of pulse therapy on different orbital findings. We, therefore, emphasized the clinical usefulness of this new assay and the importance of prospective monitoring during conventional treatment or new pharmacological treatments for GO $[19,20]$. Further prospective studies are indicated to clarify the use- fulness of this assay to assess the occurrence or progression of ophthalmopathy. Studies are also required to aid in the decision making for the treatment of GO, in the prediction of response to therapy, and in assessing recurrence of ophthalmopathy after therapy. A study on the role of aequorin TSAb on the occurrence or exacerbation of ophthalmopathy after ${ }^{131}$ I therapy for Graves' disease is being presently undertaken by our study group.

In conclusion, the aequorin TSAb assay was more sen- 
sitive than the conventional assays for diagnosing GO especially euthyroid GO. This novel TSAb assay may have a promising clinical implication in GO.

\section{Acknowledgments}

This study was supported by the Research Program of
Intractable Diseases provided by the Ministry of Health, Labour and Welfare of Japan.

\section{Disclosure}

The authors declare that they have no conflict of inter-

\section{References}

1. Bartalena L, Tanda ML (2009) Clinical practice. Graves' ophthalmopathy. N Engl J Med 360: 994-1001.

2. Bahn RS (2010) Graves' ophthalmopathy. $N$ Engl J Med 362: 726-738.

3. Bartalena L, Pinchera A, Marcocci C (2000) Management of Graves' ophthalmopathy: reality and perspectives. Endocr Rev 21: 168-199.

4. Hiromatsu Y, Eguchi H, Tani J, Kasaoka M, Teshima Y (2014) Graves' ophthalmopathy: epidemiology and natural history. Intern Med 53: 353-360.

5. Solomon DH, Chopra IJ, Chopra U, Smith FJ (1977) Identification of subgroups of euthyroid Graves' ophthalmopathy. N Engl J Med 296: 181-186.

6. Hiromatsu Y, Sato M, Tanaka K, Nonaka K, Kojima K, et al. (1992) Anti-eye muscle antibodies and hypothyroid Graves' disease: a case report. Endocrinol Jpn 39: 593 600 .

7. Hiromatsu Y, Sato M, Inoue Y, Koga M, Miyake I, et al. (1996) Localization and clinical significance of thyrotropin receptor mRNA expression in orbital fat and eye muscle tissues from patients with thyroid-associated ophthalmopathy. Thyroid 6: 553-562.

8. Noh JY, Hamada N, Inoue Y, Abe Y, Ito K, et al. (2000) Thyroid-stimulating antibody is related to Graves' ophthalmopathy, but thyrotropin-binding inhibitor immunoglobulin is related to hyperthyroidism in patients with Graves' disease. Thyroid 10: 809-813.

9. Eckstein AK, Plicht M, Lax H, Neuhäuser M, Mann K, et al. (2006) Thyrotropin receptor autoantibodies are independent risk factors for Graves' ophthalmopathy and help to predict severity and outcome of the disease. J Clin Endocrinol Metab 91: 3464-3470.

10. Lytton SD, Ponto KA, Kanitz M, Matheis N, Kohn LD, et al. (2010) A novel thyroid stimulating immunoglobulin bioassay is a functional indicator of activity and severity of Graves' orbitopathy. J Clin Endocrinol Metab 95: 2123-2131.

11. Diana T, Kahaly GJ (2018) Thyroid stimulating hormone receptor antibodies in thyroid eye disease-methodology and clinical applications. Ophthalmic Plast Reconstr Surg 34: S13-S19.

12. Araki N, Iida M, Amino N, Morita S, Ide A, et al. (2015) Rapid bioassay for detection of thyroid-stimulating antibodies using cyclic adenosine monophosphate-gated calcium channel and aequorin. Eur Thyroid J 4: 14-19.

13. Hiromatsu Y, Kojima K, Ishisaka N, Tanaka K, Sato M, et al. (1992) Role of magnetic resonance imaging in thyroidassociated ophthalmopathy: its predictive value for therapeutic outcome of immunosuppressive therapy. Thyroid 2: 299-305.

14. Japan Thyroid Association (2011) Diagnosis and management of Graves' malignant exophthalmos (Thyroid associated ophthalmopathy) v.1. (in Japanese) Available at www.japanthyroid.jp/doctor/img/basedou.pdf. Accessed August 28, 2019.

15. (1992) Classification of eye changes of Graves' disease. Thyroid 2: 235-236. Available at https://www.liebertpub.com/doi/pdf/10.1089/thy.1992.2.235 Accessed November 11, 2019.

16. Bartalena L, Baldeschi L, Dickinson A, Eckstein A, Kendall-Taylor P, et al. (2008) Consensus statement of the European Group on Graves' orbitopathy (EUGOGO) on management of GO. Eur J Endocrinol 158: 273-285.

17. Donaldson SS, Bagshaw MA, Kriss JP (1973) Supervoltage orbital radiotherapy for Graves' ophthalmopathy. $J$ Clin Endocrinol Metab 37: 276-285.

18. Eguchi H, Tani J, Hirao S, Tsuruta M, Tokubuchi I, et al. (2015) Liver dysfunction associated with intravenous methylprednisolone pulse therapy in patients with Graves' orbitopathy. Int J Endocrinol 2015: 835979.

19. Smith TJ, Kahaly GJ, Ezra DG, Fleming JC, Dailey RA, et al. (2017) Teprotumumab for thyroid-associated ophthalmopathy. N Engl J Med 376: 1748-1761.

20. Wang C, Ning Q, Jin K, Xie J, Ye J (2018) Does rituximab improve clinical outcomes of patients with thyroidassociated ophthalmopathy? A systematic review and meta-analysis. BMC Ophthalmol 18: 46. 